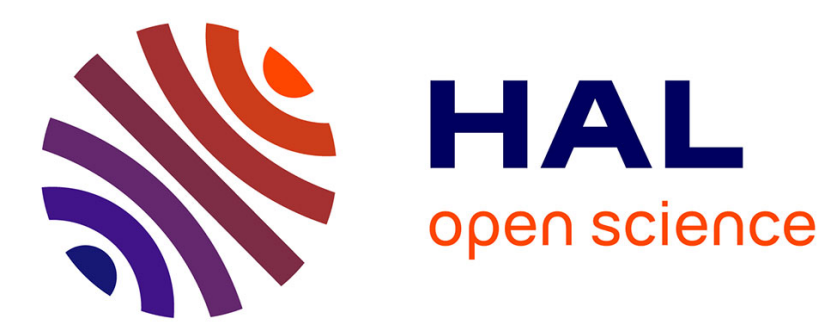

\title{
Novel quantitative indicators to characterize the protective effect of mountain forests against rockfall
}

S. Dupire, Franck Bourrier, Jean-Matthieu Monnet, Sylvain Bigot, Laurent

Borgniet, Frédéric Berger, Thomas Curt

\section{- To cite this version:}

S. Dupire, Franck Bourrier, Jean-Matthieu Monnet, Sylvain Bigot, Laurent Borgniet, et al.. Novel quantitative indicators to characterize the protective effect of mountain forests against rockfall. Ecological Indicators, 2016, 67, pp.98 - 107. 10.1016/j.ecolind.2016.02.023 . hal-01502945

\section{HAL Id: hal-01502945 \\ https://hal.science/hal-01502945}

Submitted on 6 Apr 2017

HAL is a multi-disciplinary open access archive for the deposit and dissemination of scientific research documents, whether they are published or not. The documents may come from teaching and research institutions in France or abroad, or from public or private research centers.
L'archive ouverte pluridisciplinaire HAL, est destinée au dépôt et à la diffusion de documents scientifiques de niveau recherche, publiés ou non, émanant des établissements d'enseignement et de recherche français ou étrangers, des laboratoires publics ou privés. 


\title{
Novel quantitative indicators to characterize the protective effect of mountain forests against rockfall
}

\author{
S. Dupire ${ }^{\mathrm{a}, *}$, F. Bourrier ${ }^{\mathrm{a}}$, J-M. Monnet ${ }^{\mathrm{a}}$, S. Bigot ${ }^{\mathrm{b}}$, L. Borgniet ${ }^{\mathrm{a}}$, F. Berger ${ }^{\mathrm{a}}$, T. Curt $^{\mathrm{c}}$ \\ ${ }^{a}$ Université Grenoble Alpes, Irstea, UR EMGR, 2 rue de la Papeterie-BP 76, F-38402 St-Martin-d'Hères, France \\ ${ }^{b}$ Université Grenoble Alpes, F-38402 Grenoble, France \\ ${ }^{c}$ Irstea, UR EMAX, F-13185 Aix en Provence, France
}

\begin{abstract}
Natural hazards are frequent in mountain areas where they regularly cause casualties and damages to human infrastructures. Mountain forests contribute in mitigating these hazards, in particular rockfalls. Assessing the protective effect of a forest against rockfall is a difficult task for both forest managers and rockfall experts. Accurate and simple tools are therefore required to efficiently evaluate the level of protection that results from the presence of forest.

This study defines three novel indicators to quantify the protective effect of forests against rockfalls, regarding 1) the reduction of the frequency of rockfalls, 2) the reduction of their maximum intensity, and 3) the combination of the reduction of the frequency and the energy of the rocks. The first two indicators are relevant for rockfall experts whereas the third is mostly interesting for foresters as it summarizes the protective effect of forest. The Rockyfor3D model was adapted and used to simulate rockfalls propagation on 3886 different forest stands located in all the French Alps. The results of the simulations were used to calculate the three indicators for each forest stand. Finally, the relations between the forest structures and compositions and the indicators values were investigated.

Our principal result shows that only three forest characteristics are required to accurately predict the indicators and evaluate the protective level of a forest against rockfall. The two first variables correspond to the basal area and the mean diameter at breast height (DBH) of the forest stand which are two parameters commonly used by forest managers. The third characteristic is the length of forest in the maximum slope direction which can be computed with a geographic information system (GIS). The method proposed in this study is easily reproducible and is suitable to evaluate the protective effect of European mountain forests at different scales. At local scale, the proposed indicators can enrich rockfall studies in which forests are usually set aside to simplify the evaluation. Moreover, the indicators may find direct applications with foresters by allowing them to identify the protective level of their forest and consequently to adapt their management. Finally, the indicators are convenient to perform spatial analysis and produce maps of the protective effect of mountain forests that could find many applications in land settlement or evaluation of ecosystem services.
\end{abstract}

Keywords: Indicators, Protection forest, Rockfall, Ecosystem services, 3D simulation model, Mountain forest

*Corresponding author at: Irstea, UR EMGR, 2 rue de la Papeterie-BP 76, F-38402 St-Martin-d'Hères, France
Email address: sylvain.dupire@irstea.fr (S. Dupire) 


\section{Introduction}

Forests have a prominent place in the mountain areas of the world. Mountain forests represent $23 \%$ of the global forest cover with over 9 million $\mathrm{km}^{2}$ (Price et al., 2011). They provide many goods and services essential to human life and activities. In addition to the wood resources they represent, mountain forests also constitute a reserve of biodiversity and contribute to the landscape attractiveness and the environmental quality. A significant proportion of mountain forests also protect human beings and infrastructures against natural hazards such as rockfall, snow avalanches, flash floods and soil erosion (Brang et al., 2001). For instance, in France, about 25\% of the Alpine forests are located between rock release areas and human infrastructures and may contribute to reduce rockfall damages and casualties (Toe and Berger, 2015).

An increasing number of studies have demonstrated that forests can be an efficient and cost-effective rockfall protection structure (Notaro and Paletto, 2012; Wehrli et al., 2006, 2005), especially for small mass events $\left(<5 \mathrm{~m}^{3}\right)$ (Berger et al., 2002). On forested slopes, a falling rock can impact or break a tree with two responses after the contact: the rock is either stopped or deviated (Dorren et al., 2006). Each time an impact occurs, the total energy of the rock is partially reduced (Bertrand et al., 2013; Dorren and Berger, 2006). Therefore, forests contribute to reduce both the energy and the number of rocks that threaten human lives and infrastructures (Stoffel et al., 2006).

In mountain areas, optimizing forest management to mitigate natural hazards while maintaining other ecosystem services is a long-term fundamental objective. For this purpose, several empirical target values for stand parameters (mainly tree density, basal area, tree diameter and spatial distribution) have been proposed to optimize the protective effect of forests (Perret et al., 2004; Wasser and Frehner, 1996; Gsteiger, 1993). These approaches have the advantages of being simple and directly practicable by forest managers. However they are mainly based on expert observations and are therefore insufficient to characterize and understand in details the functional processes involved in the interaction between forest and rockfalls. Recent studies proposed to use process-based rockfall modelling approaches that precisely describe rockfall trajectories including impacts against trees (Fuhr et al., 2015; Radtke et al., 2014; Stoffel et al., 2006). For instance, the RockyFor3D model has accurately predicted different rockfall patterns for several forested and non-forested sites in mountainous terrain (Dorren et al., 2006).

Until now, numerical methods have only been used on a restricted number of sites and forest stand structures. Moreover, few studies propose to use quantitative indicators to characterize the protection provided by forest (Radtke et al., 2014). However, both forest managers and civil protection agents need quantitative indicators in order to accurately assess the protective effect of a particular forest and decide whether additional civil engineering measures are needed.

The aims of this study are (1) to define novel indicators allowing practitioners to characterize the protective effect (PE) of a forest and (2) to apply them to quantify and compare the PE of the main forest structures and compositions existing in the French Alps. For this purpose, we first evaluated the importance of non-forest inputs on the propagation of the rocks simulated with Rockyfor3D. Second, we applied the model on 3886 forest plots of the French National Forest Inventory (NFI). Third, we proposed and calculated new indicators to quantify both the rockfall frequency and the rock energy reduction for each forest plot. Finally, we introduced a new practical method, based on the results of the simulations, to rank and predict the PE of the different forest structures and compositions in the French Alps.

\section{Material and methods}

Figure 1 shows the different steps of the methodology followed in this study. The different processes are detailed hereafter.

\subsection{Rockfall simulation settings}

The RockyFor3D software is one of the few rockfall simulation models (Dorren, 2015; Woltjer et al., 2008) which explicitly takes into account the protective effect (PE) of forests. Moreover, Rockyfor3D source code was made available for this study which made it possible to add new functionalities and to run the model in batch mode on a computing centre. RockyFor3D calculates trajectories of single, individually falling rocks, in three dimensions (Dorren et al., 2006). The model simulates the propagation of rocks down a slope on a rasterized digital terrain model by successive sequences of free flights through the air, rebounds on the slope surface, and impacts against trees. When a rock impacts a tree, it loses part of its kinetic energy depending on the tree type (broadleaves versus conifers), on the 


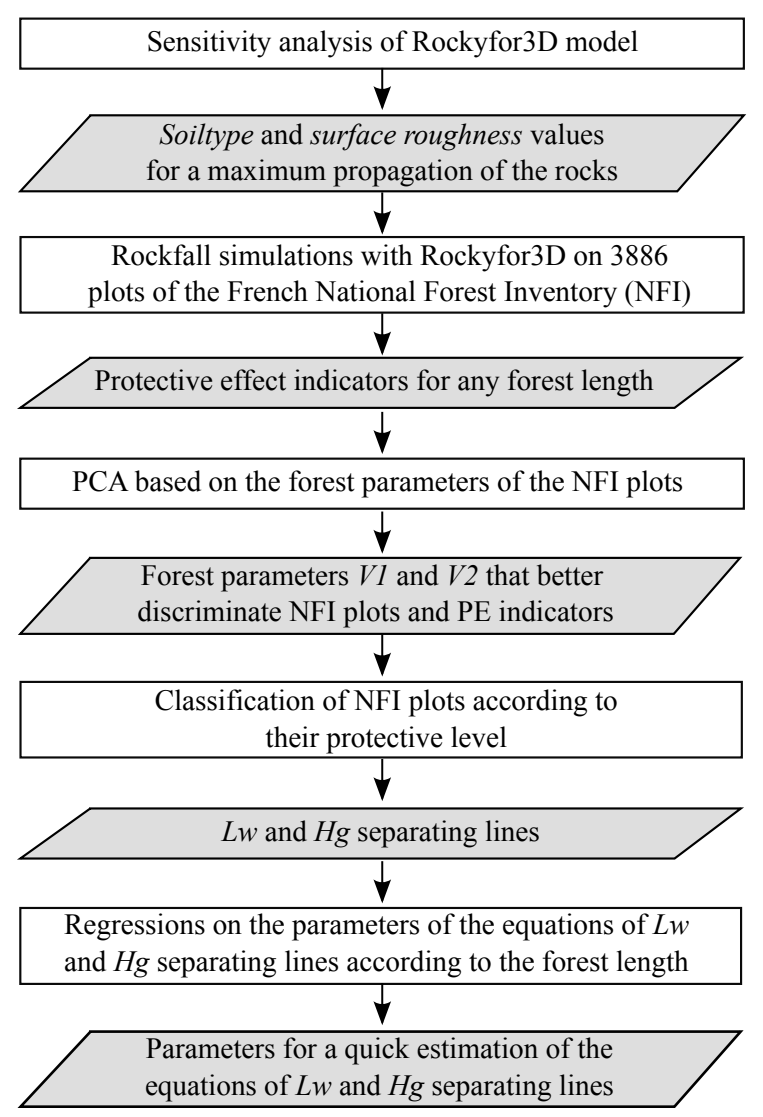

Figure 1: Steps of the study. White rectangles correspond to processes. Grey-coloured parallelograms are results.

vertical and horizontal locations of the impact on the tree, and on the rock trajectory before impact (Dorren, 2015). Rockyfor3D uses raster maps as input files that define slope surface, topography and characteristics of forest and rock. The calculation screens option allows the collection of detailed data on rockfall kinematics (mainly energy, passing height and velocity) of each rock arriving on a certain line (i.e. calculation screen) positioned along the slope surface.

In order to identify the best settings of Rockyfor3D model, a first set of simulations was run to understand the relative importance of the surface roughness and soiltype. These parameters were tested on virtual digital terrain models (DTM) with 2-m resolution, a regular slope $\alpha$ and a length $L$ of $2100 \mathrm{~m}$ (Fig. 2.a). Surface roughness was tested in the range [0 - 80] $\mathrm{cm}$ with a $1-\mathrm{cm}$ increment. In Rockyfor3D, soiltype is directly linked to the normal coefficient of restitution $R_{n}$ used in the rock rebound calculation. soiltypes 1 to 6 were tested which corresponds to $R_{n}$ values in the range [0.21 - 0.58]. Each (soiltype, surface roughness) combination was also tested on the slope range $[20-50]^{\circ}$ and with three rock volumes $\{0.5,1,5\} \mathrm{m}^{3}$. For each simulation, 15000 spherical blocks with a density of $2600 \mathrm{~kg} \cdot \mathrm{m}^{-3}$ were released on a contour line situated at the top of the virtual terrain. To ensure identical initial conditions for all simulations, we modified Rockyfor3D model to allow the parametrisation of an initial velocity and direction of the rock. Initial velocity was set to $12 \mathrm{~m} \cdot \mathrm{s}^{-1}$ and initial direction was chosen to have a normal incidence angle of $25^{\circ}$ for the first soil rebound. Those values are commonly observed in field experiments on forested slopes (Bourrier et al., 2009). Calculation screens were located every 5 meters along the slope surface in order to record the number of passing blocks depending on the distance to the release line. The ground distance from departure where $90 \%$ of the blocks were stopped $\left(d_{\text {stop }}\right)$ was used as indicator in order to compare the results of the simulations. 


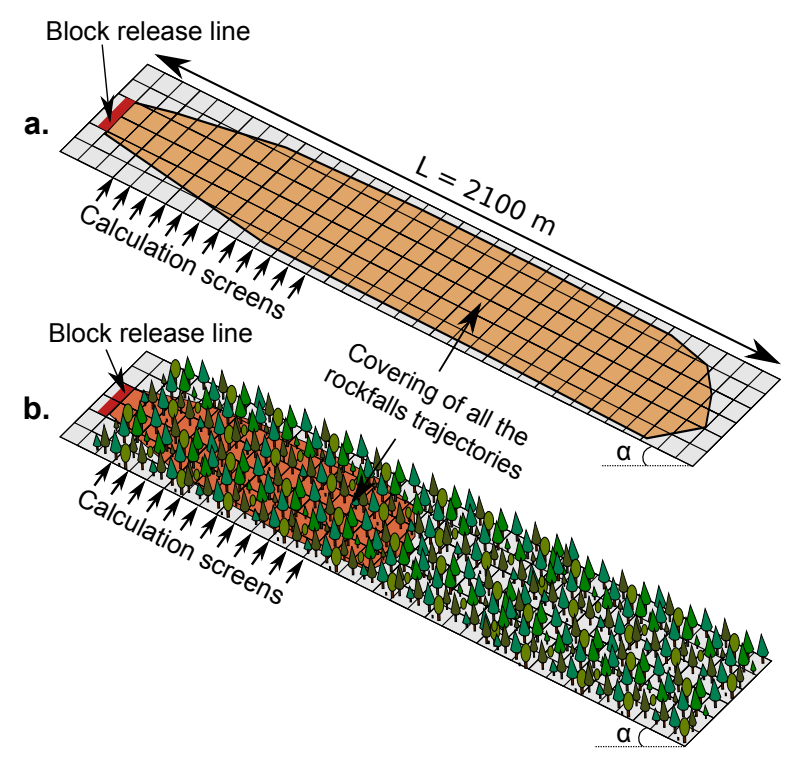

Figure 2: Virtual terrain with uniform slope $\alpha$ and length L. Calculation screens are located every $5 \mathrm{~m}$ along the non-forested (a) and forested (b) profiles.

\subsection{Source and selection of forest data}

Mountain forests present a wide diversity in terms of stand structures and composition (Price et al., 2011). Using accurate and standardized forest description for quantifying and comparing their protective effect is therefore of major importance. Since 2004, the French National Forest Inventory (NFI) is based on a systematic grid of $1 \mathrm{~km} \times 1 \mathrm{~km}$ permanent plots designed to cover the whole national forested area. Each year, $10 \%$ of the plots are measured. From 2005 to 2013, nine fractions are available with approximately 6700 forest plots each year. NFI data are collected on a system of concentric circle plots (Robert et al., 2010). Stand properties and topographic data are assessed in a 25-m radius. Tree characteristics are inventoried over a countable threshold at a diameter at breast height (DBH) of $7.5 \mathrm{~cm}$.

NFI plots contain sufficient information for rockfall modelling regarding forest inputs. However, even though soil surveys are carried out on each NFI plot, soil data are not sufficient for a faithful description of the ground surface which has to be accurately qualified in term of roughness and hardness (Bourrier et al., 2009).

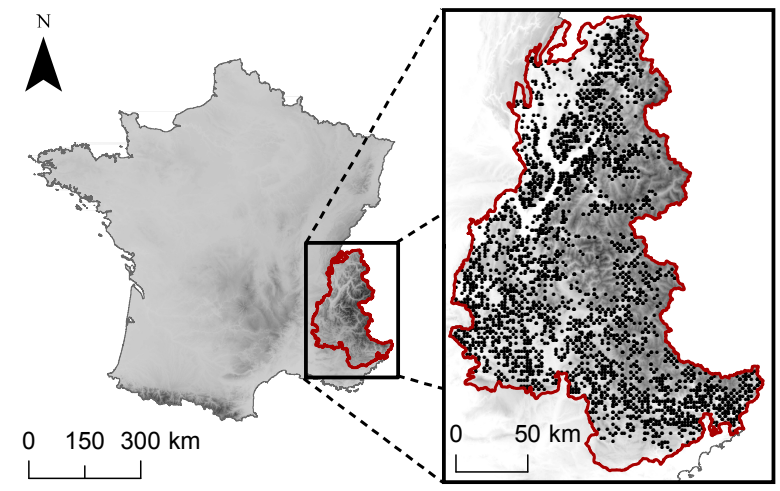

Figure 3: Map of the 3886 selected NFI Alpine plots (black dots). French Alpine region is delimited by the red line.

Our study used NFI plots for the description of both terrain slope and forest stands and structures. Only the plots located in the Alpine region (Fig. 3) with a terrain slope gradient greater than or equal to $20^{\circ}$ were used. For slope 
values less than $20^{\circ}$, rocks have a rolling mode of motion and their velocity decreases quickly (Dorren, 2003). This resulted in the selection of 3886 NFI plots measured from the period 2005-2013. Statistics about forest and slope parameters are shown in Table 1.

\begin{tabular}{llllll}
\hline & Min & $\mathbf{2 5}^{\text {th }}$ Pc & Median & $\mathbf{7 5}^{\text {th }}$ Pc & Max \\
\hline Terrain slope $\left[{ }^{\circ}\right]$ & 20 & 25 & 30 & 35 & 57 \\
Basal area $\left[\mathrm{m}^{2} \cdot \mathrm{ha}^{-1}\right]$ & 0.4 & 12.8 & 23.6 & 36.1 & 116.8 \\
Mean DBH $[\mathrm{cm}]$ & 7.6 & 13.7 & 17.3 & 23.1 & 76.7 \\
Number of stems $\left[\mathrm{ha}^{-1}\right]$ & 15 & 400 & 750 & 1200 & 5400 \\
\hline
\end{tabular}

Table 1: Statistics of terrain slope and forest stand parameters for the 3886 Alpine plots of the NFI. $25^{\text {th }} \mathrm{Pc}$ and $75^{\text {th }} \mathrm{Pc}$ are the $25^{\text {th }}$ and the $75^{\text {th }}$ percentiles respectively.

\subsection{Rockfall simulations on NFI plots}

A similar method as in section 2.1 was used for rockfall simulations on the NFI plots. For each plot, a new virtual terrain was generated and used for both forested and non-forested simulations. Each virtual terrain had a regular slope $\alpha$ corresponding to the NFI plot slope and a total length $L$ of $2100 \mathrm{~m}$ in the slope direction (Fig. 2). Calculation screens were located every 5 meters along the slope surface to register both kinetic energy and number of passing blocks according to the distance to the release line. The rock properties and initial conditions exposed above were also used for NFI plot rockfall simulation.

In order to compare and emphasize differences in the protection provided by the different stand structures and compositions, we used the same (surface roughness, soiltype) combination for all NFI plots. The sensitivity analysis carried out at section 2.1 allowed us to identify a combination that ensures a complete propagation of all the released blocks on the $2100 \mathrm{~m}$ long virtual terrain for all non-forested simulations. This combination presents two advantages. It emphasizes the protective effect of the forest and it corresponds to the worst plausible scenario where rocks cover a long distance down the slope.

Integration of forest characteristics in Rockyfor $3 \mathrm{D}$ is done using the exact locations ( $x, y$ coordinates) and the diameters of all trees on the slope surface. When a rock impacts a tree, its energy is reduced due to both energy transfer and dissipation. Equation 1 is used to determine the maximum energy reduction of the rock during an impact on a tree (Dorren and Berger, 2006).

$$
\text { max.Ered }=\text { Coeff }(\text { treetype }) \times 38.7 \times D B H^{2.31}
$$

In its original version, Rockyfor3D uses only two values for the Coeff(tree type): one for all broadleaves species and one for all conifers species. Moreover, tree type (i.e. broadleaf or conifer) is assigned randomly during the simulation according to the proportion of conifers present in each raster cell.

For the purpose of this study, Rockyfor3D was modified in order to use species values (published in Dorren and Berger, 2006) instead of broadleaves / conifers values for Coeff(tree type). Those values were made explicit for each tree to avoid random attribution. For each NFI plot, the forest was generated following two steps. First, the location of the trees in the slope surface were sampled with uniform probability until the tree density is equal to the measured one. Second, diameters and species of each tree were randomly attributed but respecting the diameter and species distributions observed on the NFI plot.

Finally, a total of six simulations per NFI plot were tested: one without forest and one with forest for each rock volume $\{0.5,1,5\} \mathrm{m}^{3}$. All the $23316(3 \times 2 \times 3886)$ simulations were computed on the Grenoble University high performance computing centre (CIMENT).

\subsection{Definition of indicators for the protective effect of forests against rockfall}

Three indicators were defined to characterize the PE of forests against rockfall. Each of them is a function of a distance $x$ corresponding to the length of the forested slope in the maximum slope direction.

First of all, forests act as a natural barrier against rockfall and trees contribute to stop some of the falling blocks in a way similar to civil engineering devices (i.e. nets or embankments) (Lambert et al., 2013). We called this first effect the "barrier effect" and quantified it as shown in equation 2. 


$$
\operatorname{BARI}(x)=100 \times\left(1-\frac{\operatorname{Nrock}_{\text {forest }}(x)}{\operatorname{Nrock}_{\text {noforest }}(x)}\right)
$$

The BARrier effect Index BARI $(x)$ of a forest of $x$ m length along the maximum slope direction is related to the number of passing rocks at distance $x$ for the simulation with forest Nrock forest $_{t}(x)$ divided by this same quantity for the simulation without forest $\operatorname{Nrock}_{\text {noforest }}(x)$. Therefore, $B A R I(x)$ is an indicator of frequency reduction of the rockfall hazard as it directly gives the percentage of rocks stopped due to the presence of forest on $x$ m along the maximum slope direction.

A second indicator was used to define the reduction of the rockfall maximum intensity under a forested slope of $x$ $\mathrm{m}$ along the maximum slope direction (equation 3).

$$
\operatorname{MIRI}(x)=100 \times\left(1-\frac{E 95_{\text {forest }}(x)}{E 95_{\text {noforest }}(x)}\right)
$$

The rockfall Maximum Intensity Reduction Index $\operatorname{MIRI}(x)$ is related to the $95^{\text {th }}$ percentile of the energy of all the passing rocks at distance $x \mathrm{~m}$ along the maximum slope direction for the simulation with forest $E 95_{\text {forest }}(x)$ divided by the same quantity for the simulation without forest $E 95_{\text {noforest }}(x)$.

These two indicators give an objective information about the residual rockfall risk relative to a forested slope in term of frequency $B A R I(x)$ and maximum intensity $\operatorname{MIRI}(x)$. These two elements are especially important for civil protection agents when deciding whether a civil protection structure has to be built in complement of forest.

The third indicator integrates both the proportion of stopped rocks and the total energy reduction of rocks due to the presence of forest. We called this effect the "overall rockfall protection" and quantified it with a third indicator as shown in equation 4.

$$
\operatorname{ORPI}(x)=100 \times\left(1-\frac{\operatorname{Ecum}_{\text {forest }}(x)}{\text { Ecum }_{\text {noforest }}(x)}\right)
$$

The Overall Rockfall Protection Index $\operatorname{ORPI}(x)$ of a forest of $x \mathrm{~m}$ length along the slope direction is related to the total energy of all the blocks passing at distance $x$ with forest $E_{c u m}$ forest $_{1}(x)$ divided by this same quantity without forest Ecum $_{\text {noforest }}(x)$.

Presently, foresters mostly use indicators similar to $B A R I(x)$ which, by definition, only regards the reduction of the rockfall frequency due to forests. Therefore, they overlook a significant effect of forest which is the reduction of the energy of the rocks each time they impact a tree. ORPI $(x)$ presents the advantage of combining both effects in one indicator. It can therefore be especially interesting for foresters that would like to take into account the protective effect of their forests in its entirety.

The three indicators have a range of values from 0 (no protective effect) to 100 (high protective effect). The calculation screens located every $5 \mathrm{~m}$ along the slope surface (Fig. 2) were used to collect the values with and without forest of Nrock, Ecum and E95. The three PE indicators were then calculated using these values on each NFI plot and for each rock volume. This resulted on $11658(3 \times 3886)$ simulations where values of the three PE indicators are known for $x \in[50-2100] \mathrm{m}$.

\subsection{Classification and prediction of the PE indicators from forest characteristics}

The previous work led us to the calculation of each PE indicator according to the forest length and the rock volume for all the NFI plots (Fig. 1).

Starting from the previous results, we first investigated the effect of the forest length along the slope direction $x$ on each PE indicator. A principal component analysis was then conducted to identify the forest parameters that better discriminate both NFI plots and PE indicators. These parameters were used to elaborate a simple classification of the NFI plots into three levels of protection. Finally, we proposed a method to predict the level of protection for each PE indicator and for any European mountain forest directly from forest characteristics. 


\subsubsection{PCA on the NFI plots}

A principal component analysis (PCA) was conducted in order to compare PE indicators with forest stand characteristics. The basal area, stem density, mean DBH, mean Coeff(tree type) and terrain slope of the 3886 NFI plots were used for the PCA. PE indicators for different rock volumes and forest length $x$ were fitted afterwards.

The PCA was used to identify the two forest stand variables ( $V 1$ and $V 2)$ that allow the best discrimination of both the NFI point cloud and the PE indicators. A Cartesian coordinate system with $V 1$ as abscissa and $V 2$ as ordinate was defined to perform the prediction of the protective effect and the classification of the NFI plots.

\subsubsection{Classification of NFI plots according to their protective effect}

NFI plots were divided randomly into two equivalent samples $A$ (calibration) and $B$ (validation) of 1943 NFI plots each. Plots of samples $A$ and $B$ were projected in the reference system defined by $V 1$ and $V 2$.

$\mathrm{PE}$ values of each plot of the sample $A$ were transformed in three levels of protection for each PE indicator. The first level corresponds to plots with PE value $\leq 50$. We qualified the PE of those forests as "Low PE" as rockfall hazard is hardly reduced. Second level corresponds to plots with PE value from 50 to 90 . We qualified the PE of those forests as "Medium PE". In this case, rockfall hazard is a least reduced by a factor 2. Last level gathers plots with a $P E$ value $\geq 90$. Those forests have a "High PE" as the rockfall component is at least reduced by a factor 10 .

For each PE indicator and each forest length $x$, we computed the equations of the straight lines $H g$ that best separate the plots of sample $A$ with PE levels equals to "Medium" and "High". Equations of the straight lines $L w$ that best separate the plots of sample $A$ with PE levels equals to "Low" and "Medium" were also computed. These equations are of type:

$$
V 2=\text { slope } \times V 1+\text { intercept }
$$

To facilitate the use of the prediction method by the practitioners, the computation was chosen to keep the same value for the intercepts of the two separating lines $L w$ and $H g$ for each couple (PE indicator, forest length). Once all the equations of the separating lines were computed, it was possible to predict the PE level of any forest type and length by locating it in the reference system defined by $V 1$ and $V 2$. For example, a forest located between the separating lines $L w$ and $H g$ will get a "Medium" PE.

To evaluate the quality of this classification, NFI plots from the sample $B$ were projected in the reference system. Their calculated PE values were transformed into PE levels and compared with the PE level obtained according to their location relatively to the separating lines $L w$ and $H g$. A contingency table was build for each PE indicator and forest length $x$ and used to calculated the accuracy of the classification. Four statistics were used to evaluate the accuracy (Congalton, 1991):

- Total accuracy: obtained by dividing the total number of correctly classified plots by the total number of plots.

- User accuracy: obtained by dividing the total number of correctly classified plots in a category by the total number of predicted plots in that category. It measures the commission error by category. In this study, we present the average user accuracy.

- Producer accuracy: obtained by dividing the total number of correctly classified plots in a category by the total number of observed plots in that category. It measures the omission error by category. In this study, we present the average producer accuracy.

- Cohen Kappa coefficient (Cohen, 1960): it reflects the difference between actual agreement and the agreement expected by chance. Kappa is always less than or equal to 1 . The agreement is generally qualified as good from 0.6 to 0.8 and very good when it is greater than 0.8 . A value of 1 indicates a perfect agreement.

\subsubsection{Prediction of the PE indicators from forest characteristics}

Linear regressions between the forest length $x$ and the slope and intercept values for both lines $H g$ and $L w$ of each PE indicator were computed. The resulting coefficients were used to draw both $\mathrm{Hg}$ and $L w$ separating lines and predict the PE levels of four different sites where empirical data on rockfall protection were available. 
The process to predict the PE levels from the separating lines is done by (1) calculating the slope and intercept values at a given forest length for both $\mathrm{Hg}$ and $\mathrm{Lw}$ lines from the coefficients of the linear regression and (2) locating the forest in the reference system defined by forest characteristics $V 1$ and $V 2$ (define at section 2.5.1) where $H g$ and $L w$ are drawn with the values previously found.

This process was applied for a first validation of the predicted PE indicators with empirical data. Predicted PE indicators were compared to PE values observed during three different rockfall experiments (Dorren et al., 2006; Doche, 1997; Jahn, 1988) and one numerical study where Rockyfor3D was calibrated according to the impact on trees observed on the field (Stoffel et al., 2006).

\section{Results}

\subsection{Importance of soil and surface parameters in Rockyfor $3 D$}
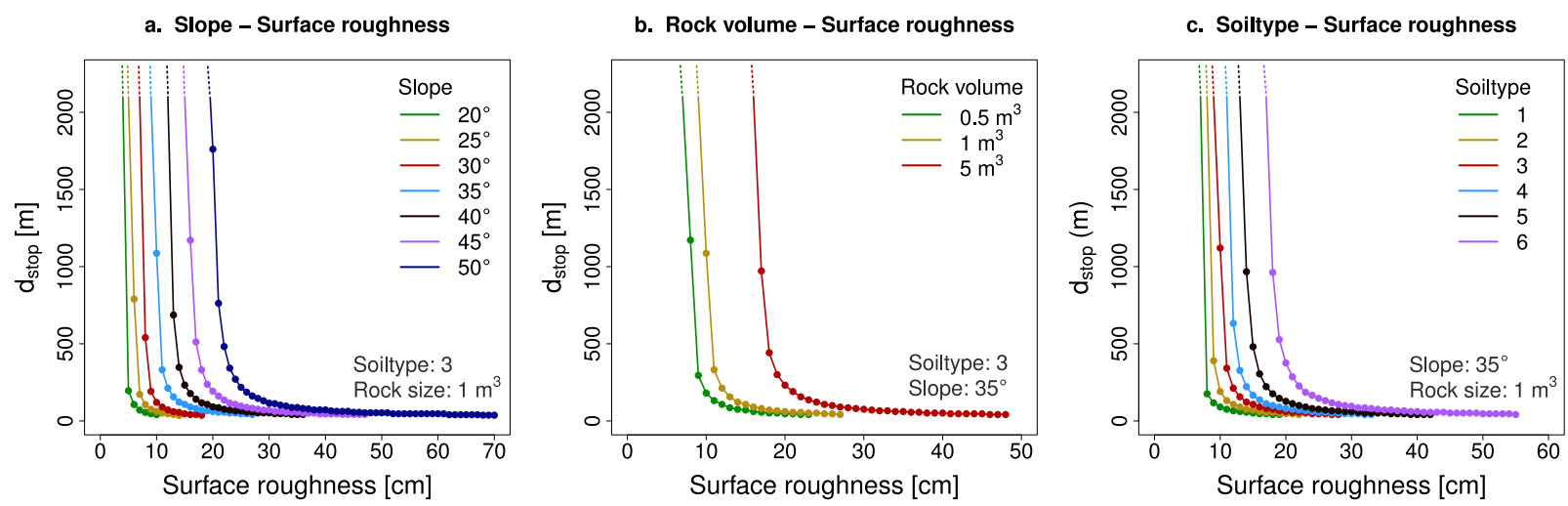

Figure 4: Distance from departure where $90 \%$ of the released blocks are stopped $\left(d_{\text {stop }}\right)$ in function of surface roughness (abscissa) and slope (a), rock volume (b) or soiltype (c).

Fig. 4 shows the influence of the surface roughness on the $d_{\text {stop }}$ value. For each combination tested, a minimum value of surface roughness exists below which none of the released block is stopped on the $2100 \mathrm{~m}$ long virtual terrain. At the opposite, a maximum value of surface roughness exists above which all the released block are stopped in the first $50 \mathrm{~m}$ of the virtual terrain. Between these minimum and maximum values, $d_{\text {stop }}$ decreases when surface roughness increases. These first simulations show that Rockyfor3D model is sensitive to surface roughness with a presence of two threshold values corresponding respectively to a complete propagation and a total stop of all the blocks. Increasing the slope, rock volume or soiltype (i.e. $R_{n}$ ) only enlarges the values taken by these two thresholds.

\subsection{Effect of the length of forested slope on the PE indicators values}

The effect of the forest length along the slope on the three PE indicators is displayed in Figure 5. The barrier effect index $B A R I(x)$, the maximum intensity reduction index $\operatorname{MIRI}(x)$ and the overall rockfall protection index $O R P I(x)$ evolve following an increasing curve to a horizontal asymptote for the long forested slopes. The range of values taken by $B A R I(x)$ is very wide. This range slightly decreases for $\operatorname{MIRI}(x)$ and significantly decreases for $\operatorname{ORPI}(x)$. BARI $(x)$ and $\operatorname{ORPI}(x)$ are higher for small rock volumes unlike $\operatorname{MIRI}(x)$ which is higher for big rock volumes.

\subsection{Principal component analysis}

The biplot of the PCA (Fig. 6) described in section 2.5 shows the relative importance of the terrain slope and forest stand parameters of the NFI plots. PE indicators values after different forest lengths are also shown. According to the PCA, the slope of the terrain has a very low contribution to the PCA $(0.2 \%$ and $0.3 \%$ on component 1 and 2 respectively). The mean value of coeff(TreeType) observed on each NFI plot has a medium contribution to the PCA ( $4.3 \%$ and $3.9 \%$ on component 1 and 2 respectively). It is slightly higher for the stem density with $0.9 \%$ on component 

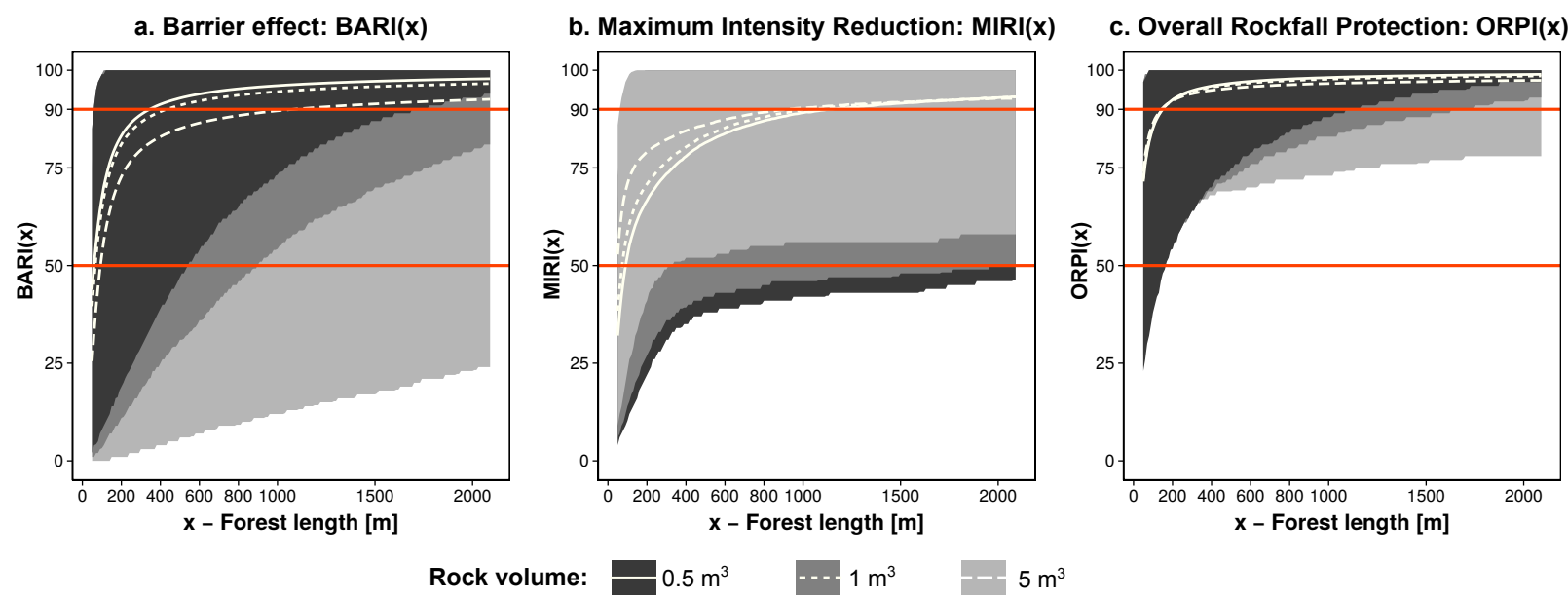

Figure 5: Effect of the forest length along the slope on the three indicators for the 3886 NFI plots. White lines show the average values for each rock volume. Gray-colored areas are placed on top of each other and represent the possible indicators values taken from the $5^{\text {th }}$ percentile to the $95^{\text {th }}$ percentile for each rock volume. Red lines represent the separating lines $L w(50)$ and $H g(90)$ between the three levels of protection used in this study.

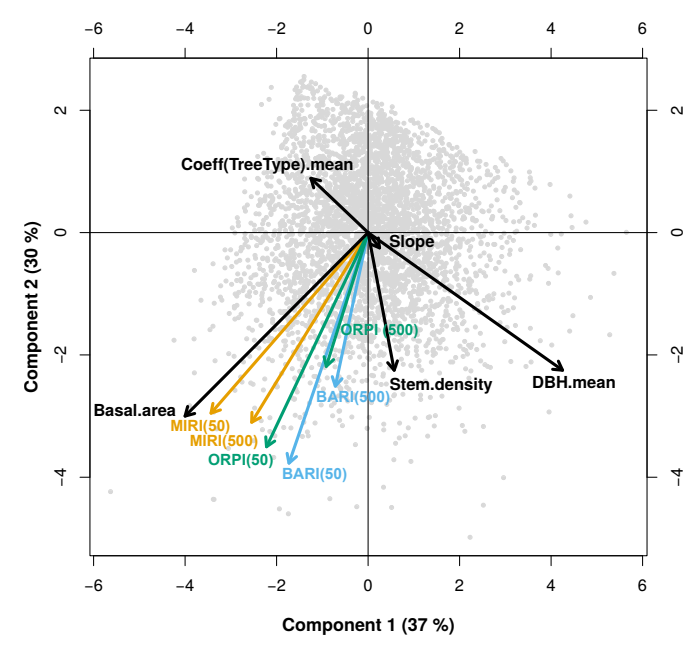

Figure 6: Biplot of the principal component analysis (PCA) based on the basal area, stem density, mean DBH, mean coefficient for tree types and terrain slope (black arrows) of the $3886 \mathrm{NFI}$ Alpine plots. PE indicators (coloured arrows) at $x=50 \mathrm{~m}$ and $x=500 \mathrm{~m}$ were fitted post hoc.

1 and $25.3 \%$ on component 2. Finally, the basal area and the mean DBH observed on NFI plots are the variables that best contribute to component 1 (44.4\% and 50.2\% respectively) and component 2 (45.1\% and $25.4 \%$ respectively). PE indicators were fitted post hoc, the biplot shows that all of them are located between basal area and mean DBH. These points led us to keep as variables $V 1$ and $V 2$ (introduced at section 2.5) the basal area and mean DBH respectively.

\subsection{Classification of NFI plots}

NFI plots were projected in the Cartesian coordinate system defined by the basal area as abscissa and the mean DBH as ordinate (Fig. 7). In this reference system, PE values evolve following a clockwise motion, this regardless of the PE indicator and the forest length $x$. PE levels "Low","Medium" and "High" were separated with two lines called 
$L w$ and $H g$ as shown in Figure 7.

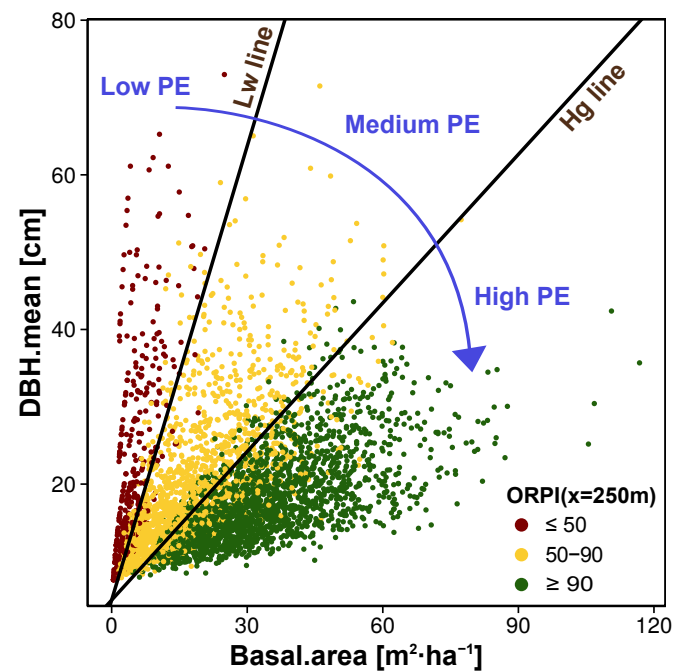

Figure 7: Illustration of the separation in three PE levels by $L w$ and $H g$ separating lines. NFI plots are projected in the Cartesian coordinate system defined with basal area as abscissa and mean DBH as ordinate. Points are coloured according to the PE levels for ORPI $(x)$ indicator at $x=250 \mathrm{~m}$ and a rock volume of $1 \mathrm{~m}^{3}$.

The quality of the partition of NFI plots with these two lines is illustrated in Figure 8. The values taken by the Cohen Kappa indicate a good agreement between observed and predicted PE level for ORPI $(x)$ and a very good agreement for $\operatorname{MIRI}(x)$ and $B A R I(x)$. Total accuracy oscillates between 0.75 and 0.95 which shows a good correlation between observed and predicted PE levels. The total accuracy is however lower for $\operatorname{BARI}(x)$. User accuracy vary from 0.7 to 0.9 which indicates a fairly good prediction. $B A R I(x)$ and $\operatorname{ORPI}(x)$ have a better user accuracy than $\operatorname{MIRI}(x)$. Finally, producer accuracy has the widest range of variation (0.62-0.95) for $\operatorname{MIRI}(x)$ and $O R P I(x)$. Producer accuracy for $B A R I(x)$ is high with a value of 0.96 .

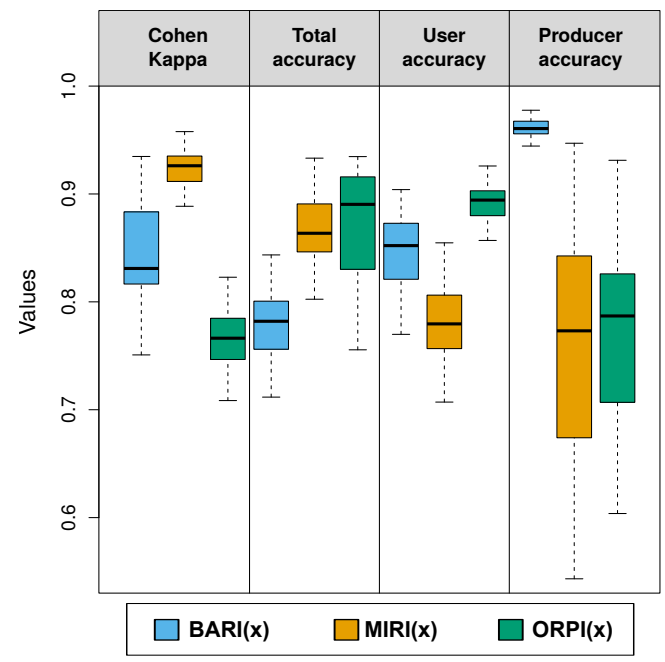

Figure 8: Quality of the partition of NFI plots in three classes of protection level for the three PE indicators. Cohen Kappa and total, user and producer accuracies were calculated from the NFI plots of sample B. This represents 615 observations for each PE indicators $(205$ values of $x \in[50$ $\mathrm{m}-2100 \mathrm{~m}] \times 3$ rock volumes) 


\subsection{Influence of the forest length on the equations of the separating lines}
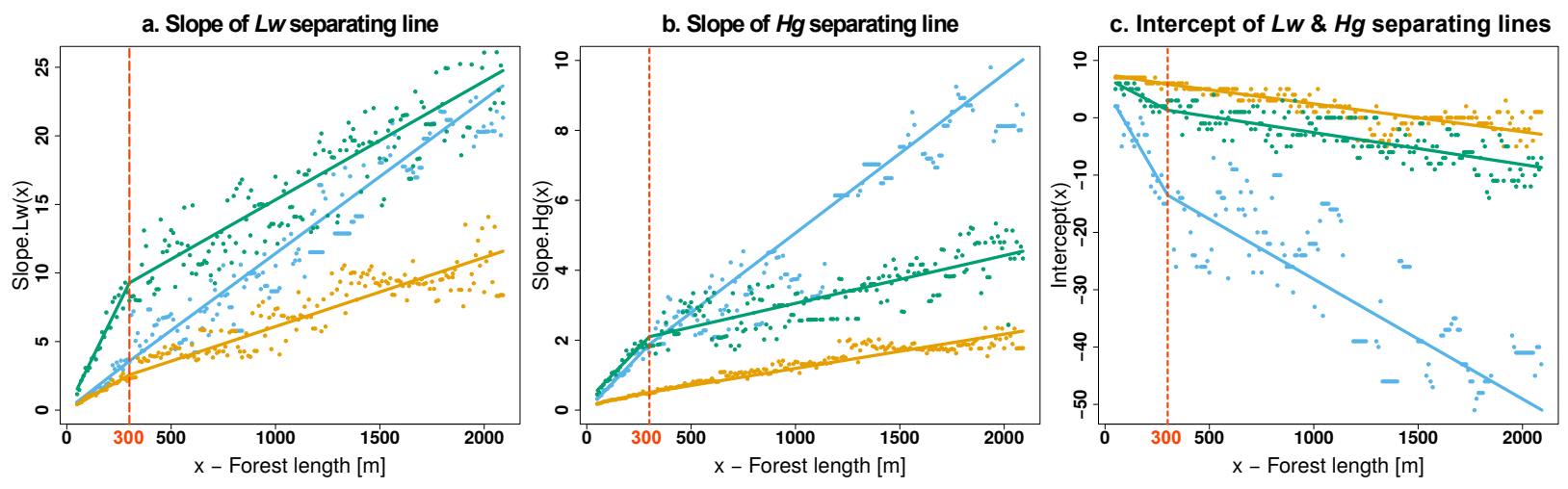

Figure 9: Influence of the length on the slopes and intercept of separating lines $H g$ and $L w$ and for a rock volume of $5 \mathrm{~m}^{3}$. The vertical dashed red line indicates the separation between short and long forest lengths.

Figure 9 shows the values of the slopes and intercept for $\mathrm{Hg}$ and $\mathrm{Lw}$ separating lines according to the forest length $x$ and for the three PE indicators. The slopes of $L w$ and $H g$ lines increase when the forest length increases, this regardless of the PE effect. In contrast, the common intercept value of $L w$ and $H g$ decreases when the forest length increases. A break can be observed around a forest length of $300 \mathrm{~m}$ which lead us to consider two classes of forest lengths: short forest length $(x \leq 300 \mathrm{~m})$ and long forest lengths $(x>300 \mathrm{~m})$.

Table 2 gives the equations for the slopes of $L w$ and $H g$ lines and their common intercept according to both the forest length $x$ and the rock volume. Coefficients are given for short forest lengths and long forest lengths. Regressions for slope- $H g$ and slope- $L w$ present a better coefficient of determination $R^{2}$ (from 0.88 and 0.99 ) than for the intercept (from 0.53 to 0.89 ).

\begin{tabular}{|c|c|c|c|c|c|c|c|c|c|c|c|c|c|c|c|c|c|c|c|}
\hline \multirow{3}{*}{$\begin{array}{c}\text { Rock } \\
\text { volume }\end{array}$} & \multirow{3}{*}{ PE } & \multicolumn{9}{|c|}{ Short forest length $(\leq 300 \mathrm{~m})$} & \multicolumn{9}{|c|}{ Long forest length $(>300 \mathrm{~m})$} \\
\hline & & \multicolumn{3}{|c|}{ Slope-Lw } & \multicolumn{3}{|c|}{ Slope-Hg } & \multicolumn{3}{|c|}{ Intercept } & \multicolumn{3}{|c|}{ Slope-Lw } & \multicolumn{3}{|c|}{ Slope-Hg } & \multicolumn{3}{|c|}{ Intercept } \\
\hline & & a & $\mathrm{b}$ & $\mathrm{R}^{2}$ & $\mathrm{a}$ & $\mathrm{b}$ & $\mathrm{R}^{2}$ & a & $\mathrm{b}$ & $\mathrm{R}^{2}$ & $\mathrm{a}$ & $\mathrm{b}$ & $\mathrm{R}^{2}$ & $\mathrm{a}$ & $\mathrm{b}$ & $\mathrm{R}^{2}$ & a & $\mathrm{b}$ & $\mathrm{R}^{2}$ \\
\hline \multirow{3}{*}{$0.5 \mathrm{~m}^{3}$} & BARI & 0.0132 & 0.00 & 0.99 & 0.0057 & 0.00 & 0.99 & .0109 & 1.68 & 0.83 & 0151 & -0.57 & 0.98 & 0.0037 & 0.61 & 0.91 & 0.0109 & 1.68 & 0.83 \\
\hline & IRI & 0041 & 0.00 & 0.98 & .0014 & 0.00 & 0.99 & 0.0035 & 7.57 & 0.53 & 0.0014 & 0.81 & 0.94 & 0.0008 & 0.17 & 0.98 & -0.0007 & 6.73 & 0.56 \\
\hline & ORPI & 0.0290 & 0.00 & 0.99 & 0.0079 & 0.00 & 0.99 & -0.0093 & 5.11 & 0.73 & 0.0187 & 3.11 & 0.92 & 0.0048 & 0.93 & 0.93 & -0.0093 & 5.11 & 0.73 \\
\hline \multirow{3}{*}{$1 \mathrm{~m}^{3}$} & BARI & 0.0139 & 0.00 & 0.98 & 0.0062 & 0.00 & 0.98 & -0.0356 & 4.70 & 0.74 & 0.0179 & -1.19 & 0.92 & 0.0056 & 0.18 & 0.95 & -0.0174 & -0.77 & 0.72 \\
\hline & MIRI & 0051 & 0.00 & 0.99 & 0.0015 & 0.00 & 0.99 & -0.0046 & 7.69 & 0.54 & 0.0020 & 0.93 & 0.90 & 0.0008 & 0.19 & 0.97 & -0.0014 & 6.74 & 0.63 \\
\hline & ORPI & 0.0277 & 0.00 & 0.98 & 0.0062 & 0.18 & 0.99 & -0.0127 & 5.15 & 0.86 & 0.0159 & 3.56 & 0.95 & 0.0084 & -0.48 & 0.89 & -0.0066 & 3.32 & 0.86 \\
\hline \multirow{3}{*}{$5 \mathrm{~m}^{3}$} & BARI & 0.0119 & 0.00 & 0.98 & 0.0063 & 0.00 & 0.99 & -0.0372 & 0.00 & 0.82 & 0.0112 & 0.21 & 0.92 & 0.0045 & 0.51 & 0.90 & -0.0245 & -3.81 & 0.81 \\
\hline & & 086 & 0.00 & 0.99 & 0013 & 0.13 & 0.95 & -0.0058 & 7.60 & 0.60 & 0.0050 & 1.06 & 0.93 & 0.0010 & 0.21 & 0.96 & -0.0049 & 7.31 & 0.89 \\
\hline & ORPI & 0.0309 & 0.00 & 0.99 & 0.0074 & 0.00 & 0.99 & -0.0190 & 7.02 & 0.72 & 0.0086 & 6.68 & 0.93 & 0.0013 & 1.83 & 0.86 & -0.0056 & 2.99 & 0.84 \\
\hline
\end{tabular}

Table 2: Coefficients of the linear regression $y=a \cdot x+b$ for the slopes and intercept of separating lines $H g$ and $L w$. Intercept is common to the two lines. For a better estimation, regressions have been implemented for short $(\leq 300 \mathrm{~m})$ and long $(>300 \mathrm{~m})$ forest lengths.

\subsection{Comparison with real-size experiments}

Table 3 shows the results of the comparison between PE levels predicted with our method and PE levels observed during real size experiments. For the four sites, the predicted PE levels are identical to the ones observed. 


\begin{tabular}{|c|c|c|c|c|c|c|c|c|c|c|c|}
\hline \multirow{2}{*}{ Source } & \multirow{2}{*}{ Location } & \multicolumn{6}{|c|}{ Site characteristics } & \multicolumn{2}{|c|}{ Observed PE } & \multicolumn{2}{|c|}{ Predicted PE } \\
\hline & & $\begin{array}{c}\text { Slope } \\
\text { gradient } \\
\left.{ }^{\circ}\right]\end{array}$ & $\begin{array}{c}\text { Forest } \\
\text { length } \\
{[\mathrm{m}]}\end{array}$ & $\begin{array}{c}\text { Stem } \\
\text { density } \\
{\left[\mathrm{ha}^{-1}\right]}\end{array}$ & $\begin{array}{c}\text { Basal } \\
\text { area } \\
{\left[\mathrm{m}^{2} \cdot \mathrm{ha}^{-1}\right]}\end{array}$ & $\begin{array}{c}\text { Mean } \\
\text { DBH } \\
{[\mathrm{cm}]}\end{array}$ & $\begin{array}{c}\text { Rock } \\
\text { volume } \\
{\left[\mathrm{m}^{3}\right]}\end{array}$ & BARI & MIRI & BARI & MIRI \\
\hline Doche (1997) & Vailly (FR) & 38 & 140 & 485 & 36.5 & 29.5 & $<0.5$ & $\begin{array}{c}\text { Med } \\
(66)\end{array}$ & $\begin{array}{l}\text { Med } \\
(75)\end{array}$ & Med & Med \\
\hline Stoffel et al. (2006) & Diemtigtal $(\mathrm{CH})$ & 40 & 95 & 520 & 20.1 & 22.0 & $<0.5$ & $\begin{array}{c}\text { Med } \\
(60)\end{array}$ & / & Med & / \\
\hline
\end{tabular}

Table 3: Comparison of empirical PE values and predicted PE levels on 4 different forests. The three first locations correspond to real size rockfall experiments, the fourth correspond to a site where Rockyfor3D has been calibrated according to rock impacts on trees observed on the field. For the observed protective effects both PE levels (e.g. Low, Medium and High) and PE values are given. For predicted protective effect only PE levels obtained with the equations of Table 2 are given.

\section{Discussion}

\subsection{Interests and limitations of numerical modelling in rockfall assessment}

Implementing rockfall field experiments in forested slope from different parts of the French Alps is not possible because of the implied financial and technical constraints. For these reasons, using accurate trajectory models for the rockfall hazard assessment is of major interest. Rockyfor3D has been calibrated and validated with real field experiments (Bourrier et al., 2012; Dorren et al., 2006) and used in different countries and forest stands (Radtke et al., 2014; Bourrier et al., 2009; Stoffel et al., 2006). The sensitivity analysis carried out in this study allows a better understanding of the relative importance of the different non-forest inputs of Rockyfor3D. The threshold values observed have a consistent progression as the needed surface roughness to stop $90 \%$ of the released rocks increases when the slope or the rock volume increase or when the soil dissipative capacity decreases (Fig. 4). According to our study, the surface roughness is a very sensitive parameter, however it is very hard to measured it on the field. This point is especially interesting for the user community of the model when calibrating ground surface parameters on a new site.

Only one combination of ground roughness and soiltype $(0 \mathrm{~cm}, 3)$ was used in this research. This choice was made to emphasize potential differences between forest plots while keeping a ground description that is common in mountain forests. In Rockyfor3D, harder soiltypes necessarily go along with higher ground roughness as they correspond either to a compact soil with large rock fragments (soiltype 4) or to bedrock (soiltypes 5 and 6) (Dorren, 2015). On the contrary softer soiltypes imply a lower rock propagation as rocks loose more energy during each rebound. The combination used in this study corresponds to a conservative analysis of the rockfall hazard with a maximum propagation of the rocks on the non-forested slope.

In this study, the forest input file was modified to better take into account the species distribution and their relative capacities to absorb a part of the falling rock energy (see section 2.3). This improvement fixes a major limitation of the original version of the software that occurred when studying mixed forest. Until now, the coefficient Coeff(tree type) used to determine the maximum energy a tree can absorb (Equation 1) was assigned randomly during the simulation according to the proportion of conifers present in each raster cell. For heterogeneous mixed forests (for example composed of broadleaves with small DBH and conifers with high DBH), the random attribution of the Coeff(tree type) could produce a virtual forest quite different from the reality (i.e. small DBH for conifers and high DBH for broadleaves). In this research, this point was resolved and the usage of Coeff(tree type) improved with the inclusion of one value by tree species instead of one by tree type (i.e. broadleaf or conifer).

To our knowledge, this study is the first to propose a complete overview of the interactions between rockfalls and forest on a wide diversity of forests taken across the Alps. Previous studies were only focused on a specific forest structure or composition. Radtke et al. (2014) focused their work on coppice forests of Northern Italy, Cordonnier et al. (2008) aimed at evaluating the long term protection efficiency in Norway spruce forest stands and Stoffel et al. (2006) studied three different coniferous stands across Switzerland. The recent study carried out by Fuhr et al. (2015) is the only reference where the effect of maturity (and by extension structure) of mountainous uneven aged stands is related to the protective effect of forest against rockfalls. 
Information about the spatial forest structure is not freely available in the French NFI database. Therefore, for each NFI plot, a forest stand had to be randomly generated on square areas with an equivalent size compared to the field plots. Although this issue has not been much investigated, previous studies showed that a random generation of the spatial forest structure can lead to a marginal overestimation of the PE against rockfall. Radtke et al. (2014) showed that a random spatial generation of coppice forests can lead to a small overestimation of the PE of about $10 \%$. However, this result concerns only a particular forest structure and the PE indicator used cannot be related to one of those proposed in this study. Jancke et al. (2009) also studied PE of coppice forests with an indicator close to $B A R I(x)$. Their results did not showed a significant difference between a random forest and a real forest. Recently, Monnet et al. (2016) focused on the influence of forest inputs on the estimation of the protective effect of three different forests: one coppice, one uneven-aged and one even-aged forest. They showed a marginal effect of using a randomly generated spatial forest structure compared to field inventory data.

For each NFI plot, the same stands characteristics was used on a regular virtual terrain of $2100 \mathrm{~m}$ length (Fig. 2). This produces a completely homogeneous forest stand on the entire mountainside which is not necessarily representative of the reality. Indeed, in mountain areas, forest structures and species compositions evolve following a gradient of altitude. This choice was made to emphasize PE differences between the 3886 NFI plots studied while controlling non-forest parameters. In further studies, it would be very interesting to apply the same methodology on forest structures and species distribution that evolves following an elevation gradient. However, for the smallest rock volumes $\left(\leq 1 \mathrm{~m}^{3}\right)$, our results showed that a high protective level ORPI $(x)$ was reached for the majority of NFI plot for forest length $x$ along the maximum slope direction over $200 \mathrm{~m}$ (Fig. 5). Similarly, a high barrier effect level BARI( $x$ ) was reached after $400 \mathrm{~m}$. These values are in the same order of magnitude as those observed in dendrogeomorphological studies carried out in France where the number of rock impacts on trees was measured along the slope direction (Favillier et al., 2015). Therefore, consider a homogeneous forest stand on distances shorter than $400 \mathrm{~m}$ is realistic and the results presented in this study can directly be used in such conditions.

The comparison with empirical data presented in section 3.6 shows the difficulty of implementing real-size experiments with sufficient instrumentation to get accurate information about reduction of frequency and intensity. Only three real experiments were found in the literature and two of them have information about the energy of the rocks. For practical reasons, these experiments were conducted with small rocks on short distance forested slopes. With these site configurations, the comparison with empirical data showed a good agreement. However, it was not possible to compare our predicted PE levels with observed PE values for rocks bigger than $0.5 \mathrm{~m}^{3}$ and slope longer than 190 $\mathrm{m}$.

\subsection{Interests and limitations of the protective effect indicators}

The protective effect indicators provided in previous studies (Radtke et al., 2014; Jancke et al., 2009; Berger and Dorren, 2007; Stoffel et al., 2006) are mainly focused on the reduction of the frequency of rockfall hazards and some of them can be related to $B A R I(x)$. In this study, we completed the analysis of rockfall mitigation by forests with two completely novel indicators. First, $\operatorname{MIRI}(x)$ gives the reduction of the maximum intensity which is especially interesting for rockfall specialists. Second, $\operatorname{ORPI}(x)$ synthesizes the reduction of rockfall hazard (regarding both frequency and intensity) that can be attributed to the presence of forest. Their simple definition makes their accurate calculations possible for any new site with the use of Rockyfor3D as only pre-requisite. To get a first idea of the PE of a forest, they can also be estimated under three levels of protection without running Rockyfor3D when the three forest characteristics required are known.

The values the three PE indicators according to the forest length along the maximum slope direction reflects a combination of two mechanical aspects (Fig. 5). First, the protective effect of a forest is linked with the probability that an impact between the rock and a tree occurs. This probability increases with forest length, and this especially if the number of stems is important (Dorren et al., 2005). Therefore, the overall growing trend of the three indicators is a consequence of the impact probability. Second, the values taken by the PE indicators are linked to the capacity of the trees to reduce the rock energy. The reduction of rockfall intensity $\operatorname{MIRI}(x)$ is higher for big rocks than for smaller ones (Fig. 5). For small volumes, trees are usually resistant enough to stop or deflect the rocks because of their low energy values. Consequently, for small rocks, the dominant PE effect is the barrier effect as a substantial part of the rocks are stopped when an impact occurs. On the contrary, for big volumes, either rocks are deflected or trees break under the impact. In both cases, the impact slows down the rock that loses a part of its kinetic energy. For big rocks, a succession of impacts induces a loss of the kinetic energy, therefore, the dominant PE effect is the rockfall 
intensity reduction. The link between the protective effect of forest and the combination of impact probability and tree resistance has already been mentioned (Brauner et al., 2005; Gsteiger, 1993), however it is interesting to see that the PE indicators proposed in this study are closely related to it.

The terrain slope was expected to have a major importance on the protective effect of forest but it turned out to be negligible. Indeed, the three PE indicators are calculated by comparing a forested scenario with a non-forest scenario, both of them being totally similar in terms of slope and ground characteristics. Therefore, they correspond to the proportion of hazard reduction due to the presence of forests. This way of calculating the PE indicators omits that rocks cover a longer distance and have a higher energy on steepest slopes. Thus, these indicators allow focusing on the proportion of the hazard that is reduced, even if the absolute rockfall hazard is higher on steep terrains.

Finally, the major finding of this study is the capacity to predict accurately each of the three PE indicators from only three forest characteristics: the basal area, the mean DBH (both variables including all trees with a DBH $\geq$ $7.5 \mathrm{~cm}$ ) and the forest length along the maximum slope direction. Moreover, the proposed transformation of PE indicators values to three PE levels allows a quick overview of the different components of the protective effect that can be expected from a particular forest without running a rockfall model. This result could be an interesting support to update and complement the online 2-D tool RockforNET. This empirical model, developed by Berger and Dorren (2007), allows a quick assessment of the residual risk of rockfall under a forested slope. Starting from forest and profile characteristics, the algorithm uses the energy lines principle (Toppe, 1987) to return an indicator of the rockfall frequency reduction. Our results, based on 3-D simulations realised on many different forests around the Alps, could enrich this online tool by simplifying the compulsory inputs and by returning the three PE indicators $\operatorname{MIRI}(x), B A R I(x)$ and $\operatorname{ORPI}(x)$. With this update, users could benefit of additional information about both frequency and intensity of the rockfall hazard.

\subsection{Implications for forest management and rockfall hazard assessment}

Our research presents different applications for both forest managers and rockfall hazard mitigation. Rockfall specialists are not necessarily foresters and can therefore have difficulties to characterize a forest on a particular site. Our approach suggests to use only three forest variables to characterize the forest stand. The forest length can be easily measured with a map and an aerial photography. Basal area and mean DBH can be estimated with a relascope and a forestry tape on several plots distributed along a transect from the release area of the rocks to the deposit area. After this step, it is possible to locate the forest in the reference system defined by the basal area and the mean DBH (Fig. 7) and to plot $\mathrm{Hg}$ and $\mathrm{Lw}$ lines with equations of Table 2. If the forest has a high protective effect level, the civil engineering fences can be designed taking into consideration the hazard reduction due to the forest. For example, if both $\operatorname{BARI}(x)$ and $\operatorname{MIRI}(x)$ are higher than 90, the expected frequency and intensity of rockfall events is reduced by 10. Therefore, a small civil engineering fence can be considered which will have a cost significantly lower than if the fence were designed without taking into account the forest. The same process can be used for forest with a medium PE level. However, if the forest has a low PE level, it will be recommended to design civil engineering fences as if there were no forest.

The protective effect of a forest against natural hazards is one of the major input keys used in silviculture guides to adapt the management (Maroschek et al., 2014; Motta and Haudemand, 2000). However, foresters are not necessarily specialists of rockfall and need simple and accurate tools to manage their forest with the objective of maintaining the best level of protection as possible. Currently, foresters mostly use indicators similar to BARI $(x)$ which concerns, by definition, only a part of the protective effect of forests (the reduction of frequency). Therefore, they overlook a significant effect of forest which is the reduction of the energy of the rocks each time they impact a tree. ORPI( $x$ ) presents the advantage of combining both effects in one indicator. Starting from an initial stand structure, our research allows a quick evaluation of this overall rockfall protection $O R P I(x)$. If the forest has a high PE level, its management must consider maintaining this high level of protection. The table 2 gives the equations of the $H g$ lines according to the forest length for each PE indicator. It provides an important support for foresters to know the threshold values they have to respect in order to keep a high PE level or to improve it in case of a medium or low initial PE level. Moreover, the indicators are convenient to perform spatial analysis and produce different maps of the protective effect of mountain forests against rockfall. Such maps could be very interesting for foresters and public stakeholders in order to prioritize their interventions according to the level of protection of the forests of the area. 


\section{Conclusion}

This study focused on the definition of indicators to help professionals to assess the protective effect of mountain forests against rockfalls. The proposed indicators were calculated on 3886 different Alpine forests from rock trajectories simulated with the Rockyfor3D model. The relation between forest characteristics and PE indicators was investigated in order to propose a fast and simple method to predict the protective effect against rockfall of any Alpine forest without necessarily resort to rockfall simulations.

To validate our research at different scales, furthers studies could apply this method on different mountainsides subject to rockfall hazard and compare the PE levels predicted only with forest characteristics to real-size experiments or Rockyfor3D simulation results.

Finally, our study can find an application with the growing interest in the valuation of ecosystem services (Helfenstein and Kienast, 2014). Indeed, the PE indicators proposed in this paper can be used to compare the costs of civil engineering fences when they are designed with or without taking into account the forest. The difference between the cost without forest and the cost with forest represents the (substitution) value of the protective effect of the forest against rockfall which is a good sample of ecosystem services.

\section{Acknowledgement}

This work was supported by the grant $\mathrm{n}^{\circ} 2101527657$ of the Action 10 "Prévention des risques naturels et hydrauliques" of the Programme 181 "Prévention des risques" of the French Ministry of Ecology, Sustainable Development and Energy. It was also supported by the project ANR SAMCO (ANR-12-SENV-0004).

Most of the computations presented in this paper were performed using the CIMENT infrastructure (https://ciment.ujfgrenoble.fr), which is supported by the Rhône-Alpes region (GRANT CPER07_13 CIRA: http://www.ci-ra.org).

\section{References}

Berger, F., Dorren, L. K., 2007. Principles of the tool Rockfor.net for quantifying the rockfall hazard below a protection forest. Schweizerische Zeitschrift für Forstwesen 158 (6), 157-165.

Berger, F., Quetel, C., Dorren, L. K., 2002. Forest: a natural protection mean against rockfalls, but with which efficiency. In: International Congress Interpraevent. pp. 815-826.

Bertrand, D., Bourrier, F., Olmedo, I., Brun, M., Berger, F., Limam, A., 2013. Experimental and numerical dynamic analysis of a live tree stem impacted by a Charpy pendulum. International Journal of Solids and Structures 50 (10), 1689-1698.

Bourrier, F., Berger, F., Tardif, P., Dorren, L., Hungr, O., 2012. Rockfall rebound: comparison of detailed field experiments and alternative modelling approaches. Earth Surface Processes and Landforms 37 (6), 656-665.

Bourrier, F., Dorren, L., Nicot, F., Berger, F., Darve, F., 2009. Toward objective rockfall trajectory simulation using a stochastic impact model. Geomorphology 110 (3-4), 68-79.

Brang, P., Schönenberger, W., Ott, E., Gardner, B., 2001. Forests as protection from natural hazards. In: Evans, J. (Ed.), The Forests Handbook. Vol. 2. Blackwell Science Ltd., Oxford, pp. 53-81.

Brauner, M., Weinmeister, W., Agner, P., Vospernik, S., Hoesle, B., 2005. Forest management decision support for evaluating forest protection effects against rockfall. Forest Ecology and Management 207 (1-2), 75-85.

Cohen, J., 1960. A coefficient of agreement for nominal scales. Educational and Psychological Measurement 20 (1), $37-46$.

Congalton, R. G., Jul. 1991. A review of assessing the accuracy of classifications of remotely sensed data. Remote Sensing of Environment 37 (1), $35-46$.

Cordonnier, T., Courbaud, B., Berger, F., Franc, A., 2008. Permanence of resilience and protection efficiency in mountain Norway spruce forest stands: A simulation study. Forest Ecology and Management 256 (3), 347-354.

Doche, O., 1997. Etude expérimentale de chutes de blocs en forêt. Tech. Rep. 97/0898, Cemagref, Grenoble.

Dorren, L. K., 2015. Rockyfor3D (v5.2) revealed - Transparent description of the complete 3D rockfall model. ecorisQ. URL www.ecorisq.org

Dorren, L. K. A., 2003. A review of rockfall mechanics and modelling approaches. Progress in Physical Geography 27 (1), 69-87.

Dorren, L. K. A., Berger, F., 2006. Stem breakage of trees and energy dissipation during rockfall impacts. Tree Physiology 26 (1), 63-71

Dorren, L. K. A., Berger, F., le Hir, C., Mermin, E., Tardif, P., 2005. Mechanisms, effects and management implications of rockfall in forests. Forest Ecology and Management 215 (1-3), 183-195.

Dorren, L. K. A., Berger, F., Putters, U. S., 2006. Real-size experiments and 3-D simulation of rockfall on forested and non-forested slopes. Natural Hazards and Earth System Sciences 6 (1), 145-153.

Favillier, A., Lopez-Saez, J., Corona, C., Trappmann, D., Toe, D., Stoffel, M., Rovéra, G., Berger, F., 2015. Potential of two submontane broadleaved species (acer opalus, quercus pubescens) to reveal spatiotemporal patterns of rockfall activity. Geomorphology 246,35 - 47.

Fuhr, M., Bourrier, F., Cordonnier, T., 2015. Protection against rockfall along a maturity gradient in mountain forests. Forest Ecology and Management 354, 224-231. 
Gsteiger, P., 1993. Steinschlagschutzwald. ein beitrag zur abgrenzung, beurteilung und bewirtschaftung. Schweizerische Zeitschrift für Forstwesen $144,115-132$.

Helfenstein, J., Kienast, F., 2014. Ecosystem service state and trends at the regional to national level: A rapid assessment. Ecological Indicators 36, $11-18$.

Jahn, J., 1988. Entwaldung und steinschlag. In: International Congress Interpraevent 1988 in Graz. pp. 185-198.

Jancke, O., Dorren, L. K., Berger, F., Fuhr, M., Köhl, M., 2009. Implications of coppice stand characteristics on the rockfall protection function. Forest Ecology and Management 259 (1), $124-131$

Lambert, S., Bourrier, F., Toe, D., 2013. Improving three-dimensional rockfall trajectory simulation codes for assessing the efficiency of protective embankments. International Journal of Rock Mechanics and Mining Sciences 60, 26-36.

Maroschek, M., Rammer, W., Lexer, M., 2014. Using a novel assessment framework to evaluate protective functions and timber production in austrian mountain forests under climate change. Regional Environmental Change, 1-13.

Monnet, J.-M., Bourrier, F., Dupire, S., Berger, F., 2016. Suitability of airborne laser scanning for the assessment of forest protection effect against rockfall. Landslides, 1-12.

Motta, R., Haudemand, J.-C., 2000. Protective Forests and Silvicultural Stability. Mountain Research and Development 20 (2), $180-187$.

Notaro, S., Paletto, A., 2012. The economic valuation of natural hazards in mountain forests: an approach based on the replacement cost method. Journal of Forest Economics 18 (4), 318-328.

Perret, S., Dolf, F., Kienholz, H., 2004. Rockfalls into forests: Analysis and simulation of rockfall trajectories — considerations with respect to mountainous forests in Switzerland. Landslides 1 (2), 123-130.

Price, M. F., Gratzer, G., Duguma, L. A., Kohler, T., Maselli, D., Romeo, R., 2011. Mountain Forests in a Changing World - Realizing values, addressing challenges. FAO/MPS and SDC, Rome.

Radtke, A., Toe, D., Berger, F., Zerbe, S., Bourrier, F., 2014. Managing coppice forests for rockfall protection: lessons from modeling. Annals of Forest Science 71 (4), 485-494.

Robert, N., Vidal, C., Colin, A., Jean-Christophe, H., Hamza, N., Cluzeau, C., 2010. National forest inventories reports : France. In: Tomppo, E., Gschwantner, T., Lawrence, M., McRoberts, R. E. (Eds.), National Forest Inventories-Pathways for Common Reporting. Springer, pp. 207-221.

Stoffel, M., Wehrli, A., Kühne, R., Dorren, L. K. A., Perret, S., Kienholz, H., 2006. Assessing the protective effect of mountain forests against rockfall using a 3d simulation model. Forest Ecology and Management 225 (1-3), 113-122.

Toe, D., Berger, F., 2015. Regional Mapping of Forest with a Protection Function Against Rockfall. In: Lollino, G., Giordan, D., Crosta, G. B., Corominas, J., Azzam, R., Wasowski, J., Sciarra, N. (Eds.), Engineering Geology for Society and Territory. Vol. 2. Springer International Publishing, Cham, pp. 1957-1959.

Toppe, R., 1987. Terrain models-a tool for natural hazard mapping. Avalanche formation, movement and effects, IAHS Publ 162, 629-638.

Wasser, B., Frehner, M., 1996. Minimale Pflegesmassnahmen für Wälder mit Schutzfunktion. Wegleitung. Bundesamt für Umwelt, Wald und Landschaft (BUWAL), Bern.

Wehrli, A., Dorren, L. K., Berger, F., Zingg, A., Schönenberger, W., Brang, P., 2006. Modelling long-term effects of forest dynamics on the protective effect against rockfall. Forest Snow and Landscape Research 80 (1), 57-76.

Wehrli, A., Zingg, A., Bugmann, H., Huth, A., 2005. Using a forest patch model to predict the dynamics of stand structure in swiss mountain forests. Forest Ecology and Management 205 (1), 149-167.

Woltjer, M., Rammer, W., Brauner, M., Seidl, R., Mohren, G. M. J., Lexer, M. J., 2008. Coupling a $3 \mathrm{~d}$ patch model and a rockfall module to assess rockfall protection in mountain forests. Journal of Environmental Management 87 (3), 373-388. 